\title{
Repositioning Psychiatry in Medical School Curricula: a study of medical students' attitudes
}

\section{Carol Quinn[1], Rekha Hegde[2], Julie Langan-Martin[1]}

Corresponding author: Dr Carol Quinn cquinn5@nhs.net

Institution: 1. University of Glasgow, 2. NHS Lanarkshire

Categories: Curriculum Planning, Education Management and Leadership, Students/Trainees,

Undergraduate/Graduate

Received: 30/05/2020

Published: 27/08/2020

\begin{abstract}
Amidst the ongoing global recruitment crisis in psychiatry there are compelling reasons to rethink the approach to, and the content and structure of, educational and experiential opportunities for medical students and foundation doctors. Medical students' attitudes to mental illness and psychiatry have been a topic of interest for many decades. The literature identifies the undergraduate years as pivotal in the formation of attitudes towards psychiatry and it has been suggested that medical students' attitudes towards psychiatry worsen as they progress through undergraduate education. The aim of this study was to explore University of Glasgow medical students' attitudes towards psychiatry and possible reasons for not choosing psychiatry as a viable career option. An electronic survey was emailed to all medical students in October 2017. A response rate of 24.5\% ( $\mathrm{N}=324)$ was achieved which was representative across year groups one to five. The findings identified generally positive attitudes towards psychiatry as a specialty.

However, year group analysis demonstrated a declining level of interest in pursuing psychiatry as a career choice. Negative attitudes towards psychiatry clustered in common themes, namely: stigmatised views about psychiatry and people with mental illness; perceived limited exposure to psychiatry in the curriculum and for experiential learning opportunities. This paper proposes that enhanced coverage of, and exposure to, psychiatry in undergraduate and postgraduate training may provide avenues into reducing negative attitudes towards psychiatry within medicine. This in turn may support national campaigns to 'rebrand' psychiatry and reinvigorate the psychiatry core trainee (CT) supply chain.
\end{abstract}

Keywords: psychiatry; medical curricula; recruitment; training; rebranding; medical students

\section{Introduction}

Physical and mental health occur in a symbiotic relationship. The literature base is well populated by research studies on the incidence and prevalence of depression post MI and stroke, and in chronic medical conditions such as diabetes and chronic pain (Mitchell et al, 2017; Whooley, 2018). Similarly, co-morbidities such as alcoholism and anxiety disorders are strongly associated with depression and other mental health conditions (Karpyak et al., 2017; 
Moulton, Pickup and Ismail, 2015). Depression is the leading cause of disability worldwide and a major contributor to the overall global burden of disease (WHO, 2018). Psychiatrists specialise in the diagnosis and treatment of mental illness. However, the ongoing global recruitment crisis in psychiatry (Brown and Ryland, 2019, Choudry and Farooq, 2017; Crabb et al 2017; Maidment et al., 2003; Rajagopal et al., 2004), means that adequate workforce planning and retention is essential to ensure that evidence based care and treatment can be effectively delivered. Early exposure to psychiatry through experiential learning in medical school is shown to increase positive attitudes towards and interest in psychiatry as a career choice (Economou et al., 2017; Warnke et al., 2017; Petkari et al., 2018). To achieve this, however, psychiatry may require a higher profile in medical curricula, perhaps even placed as an equal partner alongside medicine and surgery.

This study explores University of Glasgow medical students' attitudes towards psychiatry as a speciality and their perceptions of exposure to, and experience of, psychiatry in the curricula. This may inform current Royal College of Psychiatry 'rebranding' initiatives and GMC influenced curricula development.

\section{Methods}

A shorter, modified version of the "Attitudes to Psychiatry" questionnaire developed by Balon and colleagues (1999) was used. This questionnaire has been used extensively by other researchers (Shankar et al., 2011) in this field to gauge medical students' attitudes to psychiatry. Ethical permission was granted by the University of Glasgow. An anonymised questionnaire (Supplementary File 1) was sent electronically to Year 1-5 medical students (N=1324) on $2 / 10 / 17$. Two electronic reminders were posted at fortnightly intervals and the survey closed on 3/11/17. Descriptive statistical analysis and cluster analysis of open comments were undertaken.

\section{Results/Analysis}

A response rate of $24.5 \%$ was achieved $(\mathrm{N}=324)$. The gender and age representation of respondents was representative of the student population in University of Glasgow Medical School at the time of the study.

Respondents represented each year group (year 1:28\% and years 2-4: 16\%, year 5: 19\%, intercalated year: 6\%). Most respondents were aged 18-25 (90\%). A large proportion of respondents had some experience of psychiatry with $54 \%$ reporting a family member or friend with a mental health disorder and $31 \%$ of respondents reporting personal experience of a mental health disorder.

In general, respondents were positive about the efficacy of treatments available for mental illnesses. For example, $74.7 \%(\mathrm{~N}=239)$ of respondents either strongly or moderately agreed that "effective psychological treatments are available". While $71.3 \%(\mathrm{~N}=228)$ either strongly or moderately disagreed with the statement that "psychiatric patients hardly ever get better," suggesting that respondents recognised the positive effect that treatment could have. The scientific basis of psychiatry as a specialty was also generally recognised by respondents, with $51.4 \%(\mathrm{~N}=165)$ either strongly or moderately agreeing with the statement "there is a good evidence base for the treatment of mental health disorders". These views were stable across the year groups surveyed.

When students were asked about choosing psychiatry as a specialty, almost half $(47.5 \%, \mathrm{~N}=152)$ of respondents strongly or moderately agreed that "psychiatry offers a good work life balance." However, about one third (36.6\%, $\mathrm{N}=117$ ) of respondents either strongly or moderately agreed with the statement that "friends and fellow students, would discourage me from choosing psychiatry as a specialty." Rate of agreement with this statement increased across the year groups from $34.4 \%(\mathrm{~N}=31)$ in year 1 to $45 \%$ in Year $5(\mathrm{~N}=27)$.

When asked directly "I am interested in a career in psychiatry", 34.4\% (N=110) of respondents either strongly or moderately agreed. However, year group analysis demonstrated a declining level of interest in pursuing psychiatry as 
a career choice from $36.7 \%(\mathrm{~N}=33)$ in year 1 to $26.7 \%(\mathrm{~N}=16)$ in year 5 . This is illustrated in Figure 1.

\section{Figure 1: Interest in Psychiatry as a career by year group}

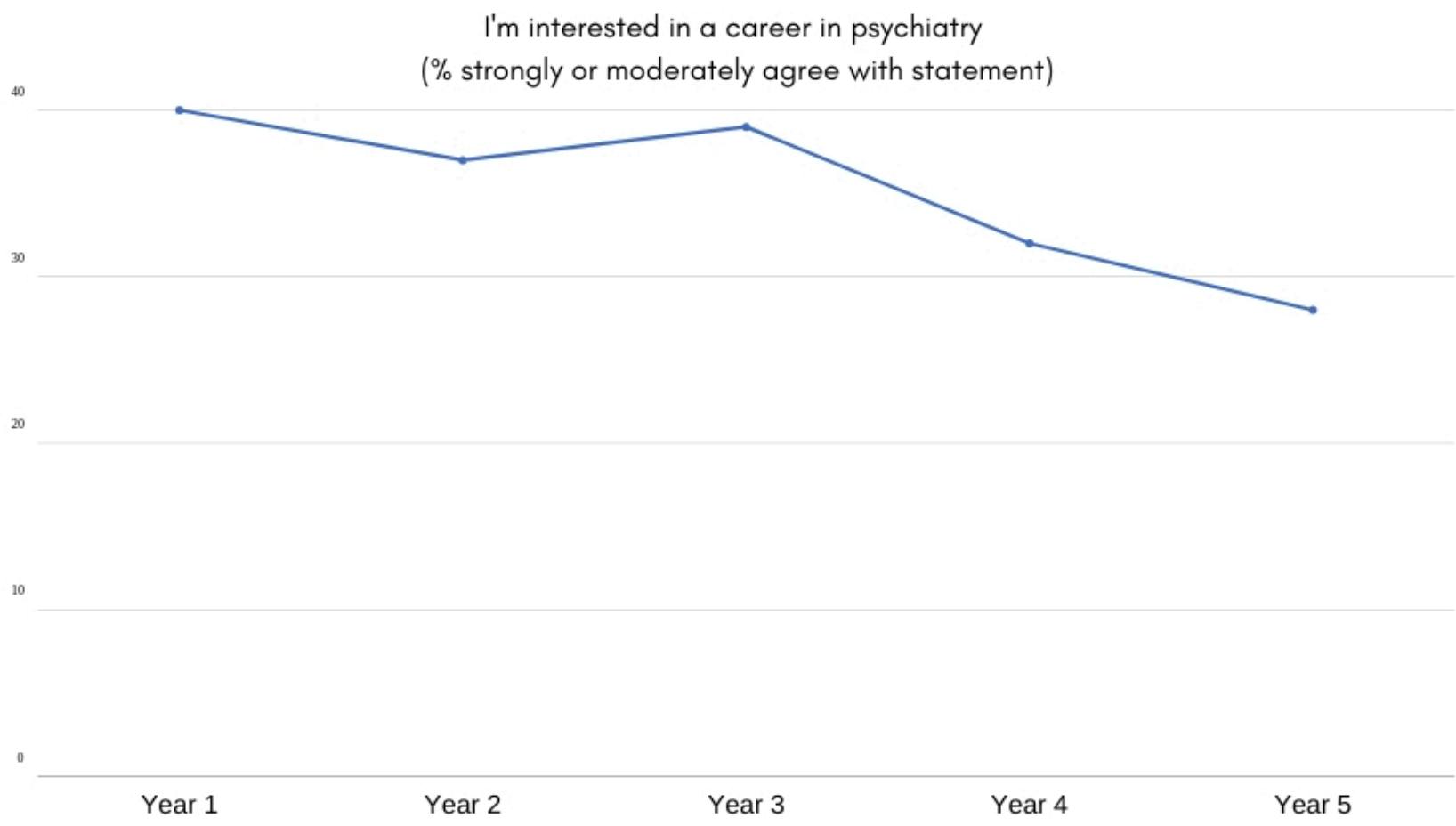

For questions relating to psychiatry education and training, respondents viewed psychiatry placements as equally useful as placements in other specialties with $85.6 \%(\mathrm{~N}=274)$ strongly or moderately agreeing with this statement. Respondents also reported that there were aware of positive role models in psychiatry, with $63.7 \%(\mathrm{~N}=204)$ strongly or moderately agreeing with this statement. However, over half of respondents $(57.5 \%, \mathrm{~N}=184)$ strongly or moderately agreed with the statement "too little time is devoted to psychiatry in the medical curriculum" and nearly three quarters $(72.7 \%, \mathrm{~N}=232)$ strongly or moderately agreed that "psychiatrists have low visibility in the general hospitals".

For questions relating to stigma in psychiatry, about one third of respondents $(35.3 \%, \mathrm{~N}=112)$ strongly or moderately agreed that they "have encountered negative perspectives of psychiatry from doctors from other specialties". There was a clear year group effect reported by respondents with $34.8 \%(\mathrm{~N}=31)$ of year 1 respondents reporting this and $75 \%(\mathrm{~N}=45)$ of year 5 students.

In an open question asking, "Why are medical students not choosing psychiatry as a specialty?" negative attitudes towards psychiatry clustered in common themes. These included: stigmatised views about psychiatry and limited exposure to psychiatry in the curriculum and experiential learning opportunities. Figure 2 captures these themes.

\section{Figure 2: Key Themes}


Majority in every year

agreed that psychiatry

was as prestigious as any

other branch of medicine
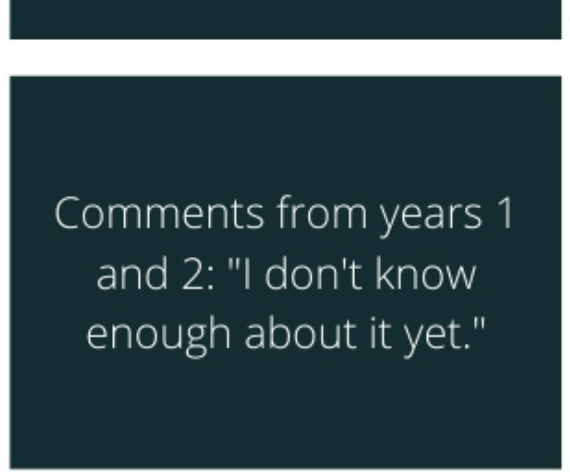
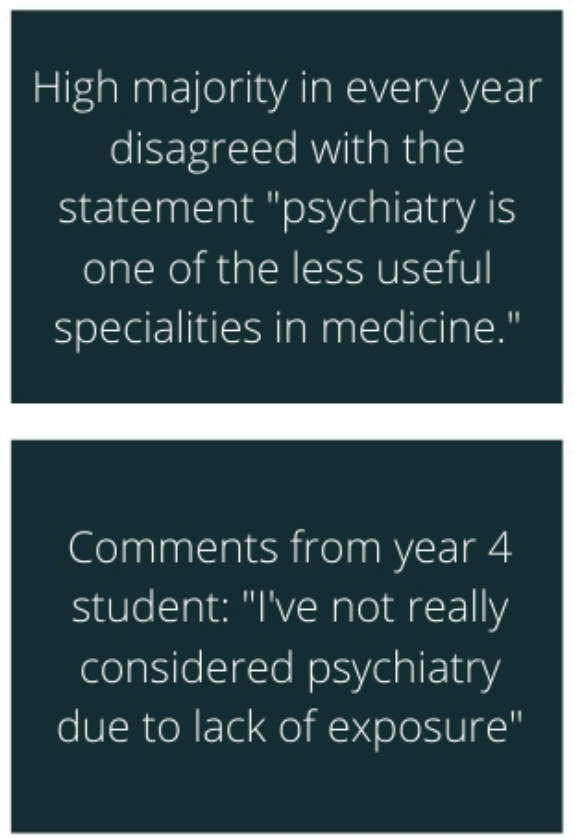

Nearly one third (32\%) of

all respondents were

interested in a career in

psychiatry, with one

quarter "neutral" on this

response.

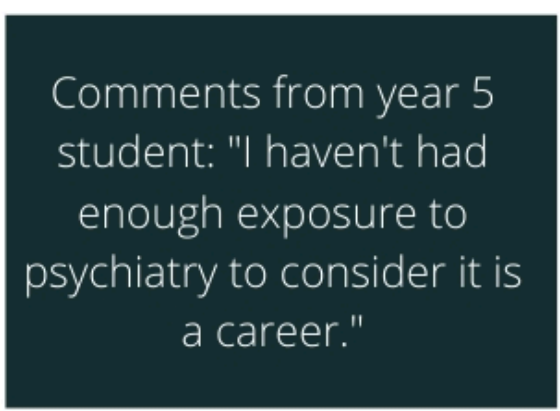

\section{Discussion}

Overall, respondents in our survey described generally positive attitudes towards psychiatry as a medical specialty, with over $70 \%$ of all year groups agreeing that psychiatry was as useful a placement in their training as any other subspecialty. However, the view of psychiatry as a scientific specialty was more mixed with just over half agreeing with this statement. The reasons for this are unclear but may be related to students' appreciation that an awareness of mental health is valuable when working with the general patient population, rather than a true appreciation of psychiatry's scientific nature.

In our cohort, almost half of respondents agreed that psychiatry offered a good work-life balance. This is an encouraging finding for promoting psychiatry as a specialty choice in light of burgeoning concerns about burn out amongst doctors. In a recent GMC study of 52,000 trainee doctors, one in four felt 'burned out' (GMC, 2017). A further study by NIHR reported that over $50 \%$ of doctors' report feeling burned out, which is associated with a two times risk to patient safety (Panagioti et al., 2018). Possibly in response to the growing awareness of burnout and its association with patient safety, the GMC has recently included questions exploring 'burnout' in the latest NTS survey.

Within the literature studies suggest that exposure to psychiatry during the early years of medical training and higher visibility in general hospitals may influence medical students' attitudes towards psychiatry (Economou et al., 2017; Petakari et al., 2018; Warnke et al., 2017). Our study supports this view, with most respondents in year groups two to five either moderately or strongly agreeing with the statement that too little time is given to psychiatry in curricula. Additionally, most respondents also reported that psychiatrists had poor visibility in the general hospital setting and so better integration of mental health wards within general hospitals and more liaison psychiatrists may help increase visibility within general hospitals and improve recruitment. Possible strategies to address these issues may involve increasing psychiatry within the undergraduate medical curriculum. The Shape of Training report (2013), which focused on five main themes and has already resulted in the GMC Generic Capability framework, rewrites of all the medical curricula and changes to medical and surgical training acknowledges that we need more 
doctors who can provide general care in broad specialties across a range of different settings (GMC, 2017). This is being driven by a growing number of people with multiple co-morbidities, an ageing population, health inequalities and increasing patient expectations. It remains to be seen whether a similar review of undergraduate medical training will take place, without which it may be difficult persuade Universities to change their curricula and give greater precedence to Psychiatry.

Stigmatised views amongst medical professionals of other medical specialties, of ten referred to as 'specialty bashing' have been identified as a possible explanation for limited interest in psychiatry, especially towards the end of medical school training. In the current study, negative attitudes about psychiatry expressed by other doctors (specialty bashing), were experienced by over half of respondents and appeared to become more problematic as students progressed through medical school year groups. The breadth of stigma towards psychiatry presents challenges for current 'rebranding' projects. Giving psychiatry greater priority in medical school curricula and foundation training programs, could help to reduce stigma formation and perpetuation, and to create pathways into addressing societal stigmatisation of mental health. It may also lead to better mental health and wellbeing within doctors and other healthcare professionals. Clearly wider anti-stigma campaigns such as Time to Change also have a role in addressing stigma at a societal level.

\section{Strengths and Limitations:}

This study adds to current knowledge by exploring possible wider, societal factors influencing medical students' attitudes to psychiatry and reasons for not choosing psychiatry as a viable career option. However, a number of limitations of our study are acknowledged. Our study was undertaken in one Scottish Medical School and so our findings are limited. Our response rate of $24.5 \%$, while comparable with other surveys, likely introduces response bias and may impact on generalisability.

\section{Conclusion}

Current efforts by the Royal College of Psychiatrists, to recruit and retain psychiatrists are encouraging. However, these efforts may require fortification from Universities and statutory government bodies in order to achieve alignment between our society's mental health needs and its ability to deliver a robust supply chain of psychiatrists. In the current climate of pyramidal shifts in postgraduate medical training and assertive 'rebranding' recruitment campaigns by the Royal College of Psychiatrists, perhaps the serendipitous repositioning of psychiatry in medical curricula would complement existing strategies.

\section{Take Home Messages}

- The majority of medical students in this study held positive attitudes towards psychiatry as a specialty and agreed with the statement that "too little time was given to psychiatry" in the curricula.

- Negative percetptions about psychiatry increased by year group.

- Enhanced coverage of psychiatry in medical school curricula may help to address stigmatised views of psychiatry and promote a more robust core training supply chain in psychiatry.

\section{Notes On Contributors}

Dr Carol Quinn is a higher trainee (ST6) in Old Age Psychiatry in NHS Tayside and an Honorary Research Fellow 
at the University of Glasgow. ORCID: https://orcid.org/0000-0003-3292-0074

Dr Rekha Hegde is a Consultant in Old Age Psychiatry in NHS Lanarkshire.

ORCID: http://orcid.org/0000-0001-6663-2076

Dr Julie Langan-Martin is a Senior Lecturer in Psychiatry and Honorary Consultant Psychiatrist at the University of Glasgow. ORCID: https://orcid.org/0000-0002-0589-3464

\section{Acknowledgements}

Thank you to the medical students at the University of Glasgow who contributed to this study.

The lead author Carol Quinn is the original author of both Figure 1 and Figure 2. There are no copyright restrictions on either Figure 1 or Figure 2.

\section{Bibliography/References}

Balon, R. (2008) 'Does a clerkship in psychiatry affect medical students' attitudes toward psychiatry?' Academic Psychiatry. 32 (2), pp. 73-75. https://doi.org/10.1176/appi.ap.32.2.73

Brown, T. and Ryland,H. (2019). 'Recruitment to Psychiatry: a global problem.' British Journal of Psychiatry International 16: 1-3. https://doi.org/10.1192/bji.2017.29

Choudry, A. and Farooq, S (2017). 'Systematic review into factors associated with the recruitment crisis in psychiatry in the UK: students', trainee ' and consultants' views.' British Journal of Psychiatry Bulletin. 4, (61). pp. 345-352. https://doi.org/10.1192/pb.bp.116.055269

Crabb, J., Barber, L. and Masson, N. (2017). 'Shrink rethink: rebranding psychiatry.' British Journal of Psychiatry. 41 (61), pp. 259-261. https://doi.org/10.1192/bjp.bp.116.197210

Economou, M., Kontoangelos, K., Peppou, L. E., Arvaniti, A., et al. (2017). ' Medical students' attitudes to mental illnesses and to psychiatry before and after the psychiatric clerkship: Training in a specialty and a general hospital.' Psychiatry Research. 258, pp. 108-115. https://doi.org/10.1016/j.psychres.2017.10.009

General Medical Council (2017) Generic Professional Capabilities Framework, May 2017, https://www.gmc-uk.org/-/media/documents/Generic_professional_capabilities_framework_0817.pdf_70417127.p df (Accessed: 20/9/2018).

Karpyak, V., Geske, J., Hall-Flavin, D., Loukianova, L., et al. (2017). 'The impact of lifetime depressionhistory on alcohol consumption in.' European Psychiatry. 41, S204. https://doi.org/10.1016/j.eurpsy.2017.01.2160

Maidment, R. L. G., Katona, M., Whitaker, E., and Katona, C. (2003). 'Carry on shrinking: career intentions and attitudes to psychiatry of prospective medical students.' Psychiatric Bulletin. 27 (1), pp. 30-32. https://doi.org/10.1192/pb.27.1.30

Mitchell, A. J., Sheth, B., Gill, J., Yadegarfar, M., et al. (2017). 'Prevalence and predictors of post-stroke mood disorders: a meta-analysis and meta-regression of depression, anxiety and adjustment disorder.' General Hospital Psychiatry. 47, pp. 48-60. https://doi.org/10.1016/j.genhosppsych.2017.04.001

Moulton, C. D., Pickup, J. C. and Ismail, K. (2015). 'The link between depression and diabetes: the search for shared mechanisms.' The Lancet Diabetes \& Endocrinology. 3 (61), pp. 461-471.

Page 16 


\section{https://doi.org/10.1016/S2213-8587(15)00134-5}

Panagioti, M., Geraghty, K., Johnson, J., Zhou, A., et al. (2018). 'Association between physician burnout and patient safety, professionalism, and patient satisfaction: A Systematic Review and Meta-analysis. JAMA Internal Medicine. 4 (10), pp.1317-1331. https://doi.org/10.1001/jamainternmed.2018.3713

Petkari, E., Gutierrez, M. A., Xavier, M., and Kustner, B. M., (2018). 'The influence of clerkship on students' stigma towards mental illness: a meta-analysis.' Medical Education. 52 (7), pp.694-704. https://doi.org/10.1111/medu.13548

Shankar, R., Laugharne, R., Pritchard, C., and Joshi, P., (2011). 'Modified Attitudes to Psychiatry Scale Created Using Principal-Components.' Academy of Psychiatry. 35 (61), pp. 360-364.

https://doi.org/10.1176/appi.ap.35.6.360

Warnke, I., Gamma, A., Buadze, M., Schliefer, R., et al. (2018). 'Predicting Medical students' current attitudes toward psychiatry, interest in psychiatry, and estimated likelihood of working in psychiatry: a cross-sectional study in four european countries.' Frontiers in Psychiatry. 9 (49). https://doi.org/10.3389/fpsyt.2018.00049

World Health Organisation, (March 2018) https://www.who.int/news-room/fact-sheets/detail/depression (Accessed: 20/9/2018).

Whooley, M.A., de Jonge, P., Vittinghoff, E., Otte, C.,et al. (2008). 'Depressive symptoms, health behaviors, and risk of cardiovascular events in patients with coronary heart disease.' JAMA. 300(20), pp.2379-2388.

https://doi.org/10.1001/jama.2008.711.

\section{Appendices}

None.

\section{Declarations}

The author has declared that there are no conflicts of interest.

This has been published under Creative Commons "CC BY 4.0" (https://creativecommons.org/licenses/by-sa/4.0/)

\section{Ethics Statement}

ESRC Framework for Research Ethics six principles were adhered to in conducting this research. A full description of the study and the intended use and publication of data was provided in the initial email to medical students. Informed consent was assumed from respondents who completed the questionnaire on a voluntary basis. Anonymity was assured as no personal details other than sex and year of study was recorded. The data was collated by the same researcher. Full ethics approval was provided by the University of Glasgow Ethics Committee 200160162 (Draft D1500539789974).

\section{External Funding}

This article has not had any External Funding 
MedEdPublish: rapid, post-publication, peer-reviewed articles on healthcare professions' education. For more information please visit www.mededpublish.org or contact mededpublish@dundee.ac.uk. 